\title{
MORPHING ACTIVE CONTOURS: A GEOMETRIC APPROACH TO TOPOLOGY-INDEPENDENT IMAGE SEGMENTATION AND TRACKING
}

\author{
Marcelo Bertalmio \\ Institute of Electrical Engineering \\ Universidad de la Republica \\ Montevideo, Uruguay \\ Guillermo Sapiro \\ Electrical and Computer Engineering \\ University of Minnesota \\ Minneapolis, MN 55455 \\ guille@ece.umn.edu \\ Gregory Randall \\ Institute of Electrical Engineering \\ Universidad de la Republica \\ Montevideo, Uruguay
}

\begin{abstract}
A method for deforming curves in a given image to a desired position in a second image is introduced in this paper. The algorithm is based on deforming the first image toward the second one via a partial differential equation, while tracking the deformation of the curves of interest in the first image with an additional, coupled, partial differential equation. The technique can be applied to abject tracking and slice-by-slice segmentation of 3D data. The topology of the deforming curve can change, without any special topology handling procedures added to the scheme. This permits for example the automatic tracking of scenes where, due to occlusions, the topology of the objects of interest changes from frame to frame.
\end{abstract}

Key words: Partial differential equations, curve evolution, morphing, segmentation, interpolation, tracking, topology.

\section{INTRODUCTION}

In a large number of applications, we can use information from one or more images to perform some operation in an additional image. Examples of this are given in Figure 1. On the top row we have two consecutive slices of a 3D image obtained from electronic microscopy. We can for example use a given segmentation of the image on the left to drive the segmentation of the next slice, the image on the right. On the bottom row we see two consecutive frames of a video sequence. Once again, we can use the image on the left to track the object in the image on the right. These are the type of problems we address in this paper.

THIS WORK WAS PARTIALLY SUPPORTED BY NSFLIS, BY THE MATH, COMPUTER, AND INFORMATION SCIENCES DIVISION AT ONR, AND BY AN ONR YOUNG INVESTIGATOR AWARD.
Our approach is based on deforming the contours of interest from the first image toward the desired place in the second one. More specifically, we use a system of coupled partial differential equations to achieve this (coupled PDE's have already been used in the past to address other image processing tasks, see $[11,12]$ and references therein). The first partial differential equation (PDE) deforms the first image toward the second one. The additional PDE is driven by the deformation velocity of the first one, and it deforms the curves marking the segmentation in the first image toward the desired segmentation in the second one. This last deformation is implemented using the level-sets numerical scheme developed in [9], allowing for changes in the topology of the deforming curve. That is, if the objects of interest split or merge from the first image to the second one, these topology changes are automatically handled by the algorithm. This means that we will be able to track scenes where objects split and merge, due to occlusions for example, and also to segment $3 \mathrm{D}$ medical data where the slices contain cuts with different topology.

\section{THE ALGORITHM}

\subsection{Basic curve evolution}

Let $\mathcal{C}(p, 0): \mathbb{R} \rightarrow \mathbb{R}^{2}$ be a set of closed curves. Assume these curves deform "in time" according to

$$
\frac{\partial \mathcal{C}(p, t)}{\partial t}=\beta \overrightarrow{\mathcal{N}}
$$

where $\beta$ is a given velocity and $\overrightarrow{\mathcal{N}}$ the inner unit normal to $\mathcal{C}(p, t)$. Let's now assume that $\mathcal{C}$ is the level-set (isophote) of a given function $u: \mathbb{R}^{2} \rightarrow \mathbb{R}$. Then, in order to represent the evolution of $\mathcal{C}$ according to (1), we have to deform $u$ 
according to

$$
\frac{\partial u}{\partial t}=\beta\|\nabla u\|,
$$

where $\beta$ is computed at the level-sets of $u$. This is the formulation introduced by Osher and Sethian [9] to implement curve evolution flows of the type of (1). This implementation has several advantages over a direct discretization of (1). Probably the main advantage is that changes in the topology of $\mathcal{C}(p, t)$ are automatically handled when evolving $u$, that is, there is no need for any special tracking of the topology of the level-sets; see [9] for details on this and the exact discretization of (2). This has motivated the use of this formulation for a large number of applications, including shape from shading, segmentation, mathematical morphology, stereo, and regularization.

\subsection{Morphing active contours}

Let $\mathcal{I}_{1}(x, y, 0): \mathbb{R}^{2} \rightarrow \mathbb{R}$ be the current frame (or slice), where we have already segmented the object of interest. The boundary of this object is given by $\mathcal{C}_{\mathcal{I}_{1}}(p, 0): \mathbb{R} \rightarrow \mathbb{R}^{2}$. Let $\mathcal{I}_{2}(x, y, 0): \mathbb{R}^{2} \rightarrow \mathbb{R}$ be the image of the next frame, where we have to detect the new position of the object originally given by $\mathcal{C}_{\mathcal{I}_{1}}(p, 0)$ in $\mathcal{I}_{1}(x, y, 0)$. Let us define a function $u(x, y, 0): \mathbb{R}^{2} \rightarrow \mathbb{R}$, such that its zero level set is the curve $\mathcal{C}_{\mathcal{I}_{1}}(p, 0)$. This function can be for example the signed distance function from $\mathcal{C}_{\mathcal{T}_{1}}(p, 0)$. With these functions as initial conditions, we define the following system of coupled evolution equations ( $t$ stands for the marching variable):

$$
\begin{aligned}
\frac{\partial \mathcal{I}_{1}(x, y, t)}{\partial t} & =\left(\mathcal{I}_{1}(x, y, t)-\mathcal{I}_{2}(x, y, 0)\right) \| \nabla \mathcal{I}_{1}(x, y, t)(\boldsymbol{\beta}) \\
\frac{\partial u(x, y, t)}{\partial t} & =\left(\mathcal{I}_{1}(x, y, t)-\mathcal{I}_{2}(x, y, 0)\right)\|\nabla u(x, y, t)\|
\end{aligned}
$$

In this system, $\mathcal{I}_{1}$ is evolving toward $\mathcal{I}_{2}$, and according to the description in the previous section, all the level sets of both $\mathcal{I}_{1}$ and $u$ are evolving with the image dependent velocity $\mathcal{I}_{1}(x, y, t)-\mathcal{I}_{2}(x, y, 0)$. More specifically, the zero level-set of $u$, which originally was positioned at the boundary of the object of interest in the current frame $\left(\mathcal{I}_{1}(x, y, 0)\right)$, follows the deformation that is moving $\mathcal{I}_{1}$ toward the next frame, $\mathcal{I}_{2}$. That is, $u$ is used to follow the deformation of the curve of interest, $\mathcal{C}_{\mathcal{I}_{1}}(p, t)$, in $\mathcal{I}_{1}(x, y, t)$. Note that in general $\mathcal{C}_{\mathcal{I}_{1}}(p, t)$, the boundary of the object of interest, will not be a level set of $\mathcal{I}_{1}(x, y, t)$ (this will happen, for example, in the case of morphing binary images; see comment below).

Eliminating the case where $\left\|\nabla \mathcal{I}_{1}(x, y, t)\right\|=0,{ }^{1}$ the steady state of $(3)$ is obtained when $\mathcal{I}_{1}(x, y, \infty)=\mathcal{I}_{2}(x, y, 0)$ that is, when the current frame has been totally deformed toward the next one. This is the basic system we propose to track from frame to frame in a video sequence and to segment from slice to slice in a 3D image.

For images with large variations of gray values inside and outside the objects delimited by $\mathcal{C}_{\mathcal{I}_{1}}(p, 0)$, we found helpful to replace $\mathcal{I}_{1}(x, y, 0)$ by the average values inside and outside $\mathcal{C}_{\mathcal{I}_{1}}(p, 0)$. This makes the boundary of the desired

\footnotetext{
${ }^{1}$ Although it is possible to find pathological situations where this can cause the flow to stop, we haven't encountered any in the large amount of examples we tested.
}

object a level-set of the modified image. This was done for the examples in Figure 2.

The complete model is obtained by multiplying the velocity in the evolution of $u$ by the term

$$
\operatorname{sign}\left(\nabla I_{1} * \nabla u\right)
$$

This allows for the concavities of $u$ to differ from those of $I_{1}$, eliminates the need of the averaging described above, and also eliminates the need to have all values outside (inside) the curve of interest grater (smaller) than the values inside (outside) of it.

\section{EXAMPLES}

The first example of our algorithm is presented in Figure 2. This figure shows an image of a neuron from the central nervous system. This image was obtained via electronic microscopy (EM). After the neuron is identified, it is marked via the injection of a color fluid. Then, a portion of the tissue is extracted, and after some processing, it is cut into thin slices and observed and captured via the EM system. The figure shows the output of the EM after some simple post-processing, mainly composed by contrast enhancement. The goal of the biologist is to obtain a three dimensional reconstruction of this neuron. As we observe from these examples, the EM images are very noisy, and the boundaries of the neuron are not easy to identify. Segmenting the neuron is then a difficult task. Using active contours techniques as those in $[3,4,6,7,8,13,14]$ normally fail with this type of images. Since the variation between consecutive slices is not big, we can use the segmentation obtained for the first slice (segmentation obtained either manually or with the technique describe in [15]), to drive the segmentation of the next one. One could argue that we could also use this segmentation to initialize the active contours mentioned above. We still encounter a number of difficulties with this approach: 1- The deforming curve still gets attracted to local minima, and often fails to detect the neuron; 2- Those algorithms normally deform either inwards or outwards (mainly due to the presence of balloon-type forces), while the boundary curve corresponding to the first image is in general neither inside nor outside of the object in the second image. Therefore, even if the image is not noisy, special techniques need to be developed and implemented to direct different points of the curve toward different directions.

The top-left image in Figure 2 shows the contour obtained from the previous slice and the one derived from it and the system (3) for the current one. The next images, from left to right and top to bottom, show consecutive slices automatically segmented after the segmentation of the first slice was obtained. Before processing for segmentation, the images are regularized using anisotropic diffusion $[1,2,10]$.

Figure 3 shows an example for object tracking. Once again, the algorithm detects the moving object without any difficulty. Other algorithms for tracking in video sequences, based on the general geodesic framework introduced in $[3,4]$ can be found in [5]. In contrast with our approach, this scheme is based on a unique PDE, deforming the curve toward a (local) geodesic curve. The algorithm is again very sensible to spatial and temporal noisy gradients. 
Figure 4 shows and additional tracking example, where the topology of the tracked boundaries changes.

\section{CONCLUDING REMARKS}

In this paper we have presented a system of coupled PDE's used for image segmentation and tracking. The first PDE deforms the first frame (or slice) toward the second one, while the additional PDE tracks the deformation of the curves of interest in the first image until they converge to the desired position in the second one.

There are a number of directions to continue the framework describe in this paper. First of all, although the technique here presented is less sensible than active contours to noisy edges, it is more sensible to changes in absolute grayvalue, as those obtained from changes in illumination (although such changes didn't affect the performance in Figure 3 ). One possible way to address this issue is to normalize the histograms of the consecutive frames. This was added to the algorithm and it is described in our extended report.

We have also applied this algorithm for 3D morphing, and for MRI visualization, and the results can be observed in our extended report.

The use of singular value decomposition and principal components analysis became very popular in computer vision and image processing in the past years. The basic idea is to represent a given event as a linear combination of principal components from learned events. We can see the technique here described as a first step toward the deformation of principal components. That is, we can look at the curve obtained from the current slice as a principal component. We are currently investigating the extension of this technique to the deformation of a number of principal components, thereby representing a given event as a combination of deformed learned principal components. The deformations will be obtained as a system of coupled PDE's.

The equations introduced in this paper are basically "short in memory," that is, only the present frame is used to segment the next one. We can incorporate past information to these equations, in the form of optical flow or Kalman filtering, in order to improve the detection results. This will be the subject of further study as well.

We are also studying the extension of our previous theoretical results for the system of coupled PDE's presented in this paper.

\section{Acknowledgments}

The images of the hand used for Figure 3 were provided by Dr. Michael Black from Xerox PARC.

\section{REFERENCES}

[1] L. Alvarez, P. L. Lions, and J. M. Morel, "Image selective smoothing and edge detection by nonlinear diffusion," SIAM J. Numer. Anal. 29, pp. 845-866, 1992.

[2] M. Black, G. Sapiro, D. Marimont, and D. Heeger, "Robust anisotropic diffusion," IEEE Trans. Image Processing, March 1998.
[3] V. Caselles, R. Kimmel, and G. Sapiro, "Geodesic active contours," Proc. Int. Conf. Comp. Vision '95, Cambridge, June 1995.

[4] V. Caselles, R. Kimmel, and G. Sapiro, "Geodesic active contours," International Journal of Computer Vision 22:1, pp. 61-79, 1997.

[5] N. Paragios and R. Deriche, "A PDE-based level-set approach for detection and tracking of moving objects," Proc. Int. Conf. Comp. Vision '98, Bombay, India, January 1998.

[6] M. Kass, A. Witkin, and D. Terzopoulos, "Snakes: Active contour models," International Journal of Computer Vision 1, pp. 321-331, 1988.

[7] S. Kichenassamy, A. Kumar, P. Olver, A. Tannenbaum, and A. Yezzi, "Conformal curvature flows: from phase transitions to active vision," Archive for Rational Mechanics and Analysis 134, pp. 275-301, 1996.

[8] R. Malladi, J. A. Sethian and B. C. Vemuri, "Shape modeling with front propagation: A level set approach," IEEE-PAMI 17, pp. 158-175, 1995.

[9] S. J. Osher and J. A. Sethian, "Fronts propagation with curvature dependent speed: Algorithms based on Hamilton-Jacobi formulations," Journal of Computational Physics 79, pp. 12-49, 1988.

[10] P. Perona and J. Malik, "Scale-space and edge detection using anisotropic diffusion," IEEE-PAMI 12, pp. 629-639, 1990.

[11] M. Proesmans, E. Pauwels, and L. van Gool, "Coupled geometry-driven diffusion equations for low-level vision," in [12].

[12] B. Romeny, Editor, Geometry Driven Diffusion in Computer Vision, Kluwer, 1994.

[13] G. Sapiro, "Color snakes," Computer Vision and Image Understanding 68:2, pp. 247-253, 1997.

[14] D. Terzopoulos, A. Witkin, and M. Kass, "Constraints on deformable models: Recovering 3D shape and nonrigid motions," $A I \mathbf{3 6}, 1988$.

[15] L. Vazquez, G. Sapiro, and G. Randall, "Segmenting neurons in electronic microscopy via geometric tracing," Proc. IEEE ICIP, Chicago, October 1998. 


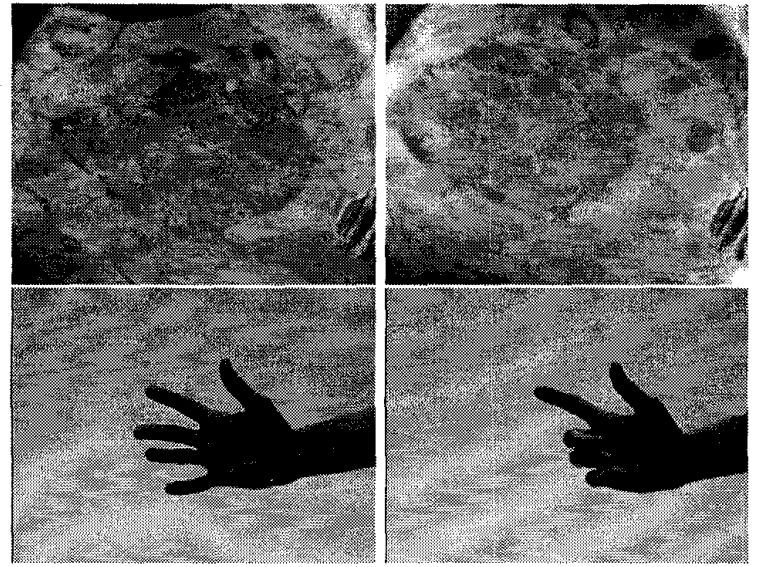

Figure 1: Examples of the problems addressed in this paper. On the top row we see two consecutive slices of a $3 D$ image obtained with electronic microscopy. The segmentation on the left can be used to drive the segmentation of the image on the right. On the bottom row we see two consecutive frames of a video sequence. The image on the left is used to track the object in the image on the right.

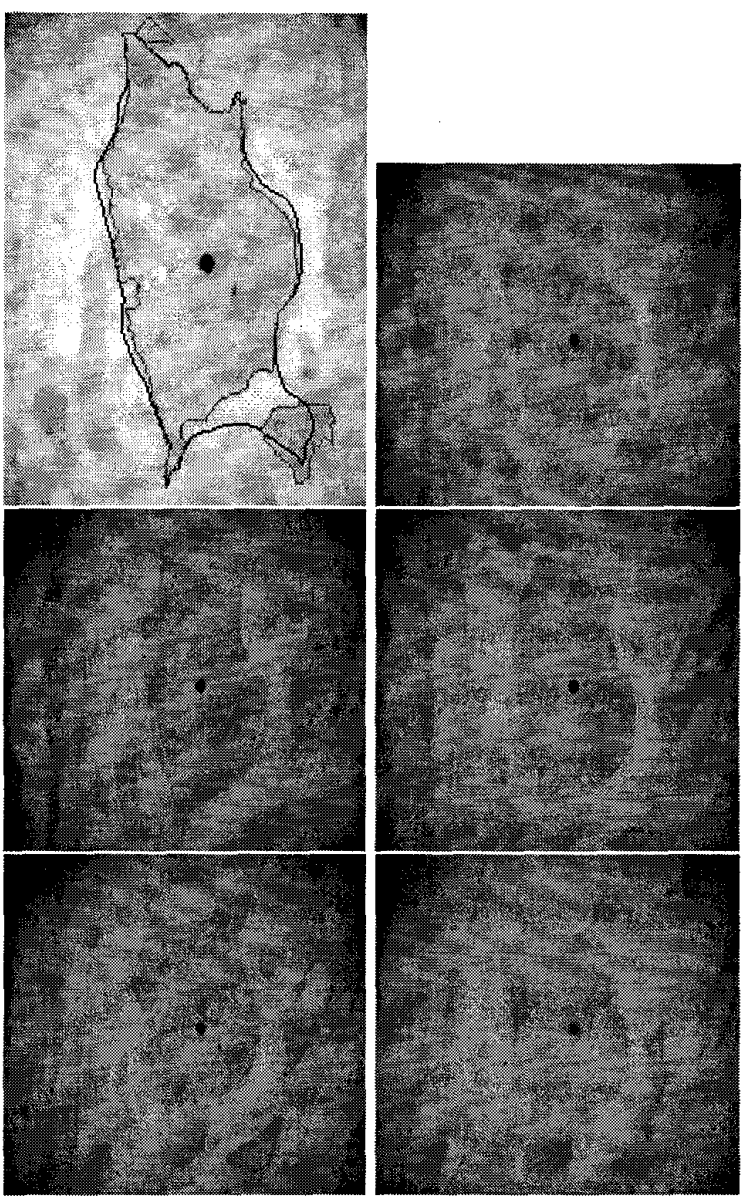

Figure 2: Example of the morphing active contours for segmenting EM. The top-left image shows in black the contour obtained from the previous slice and in red the one derived from it and the system (3) for the current one. The next images, from left to right and top to bottom, show consecutive slices automatically segmented after the segmentation of the first slice was obtained. (This is a color figure.) 

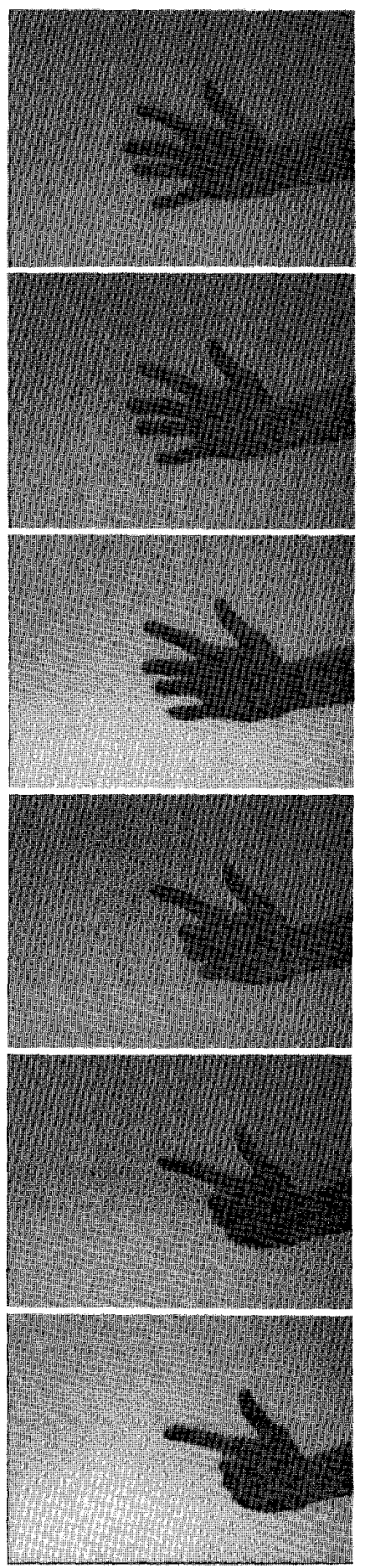

Figure 3: Tracking with the morphing active contours (green lines, from top to bottom). The 1st segmentation was done by hand, while the others where obtained with the system proposed in this paper. (This is a color figure.)

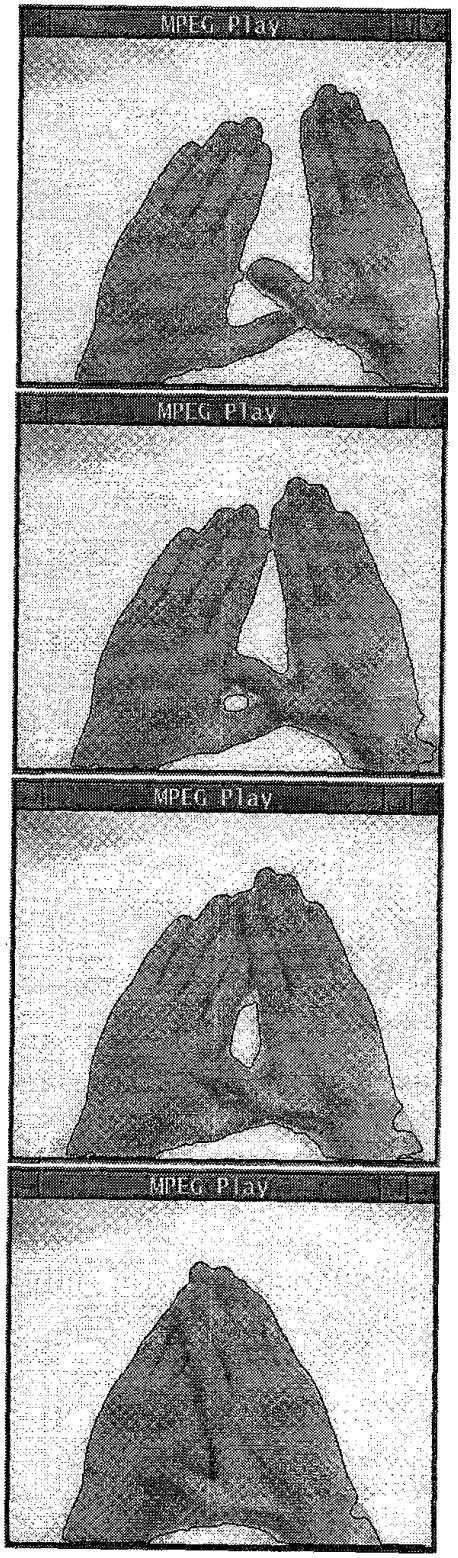

Figure 4: Tracking with the morphing active contours $\mathrm{Ob}$ serve how changes in topology are automatically tracked. (This is a color figure.) 